\title{
Difference frequency generation in topological semimetals
}

\author{
F. de Juan $\odot,{ }^{1,2}$ Y. Zhang, ${ }^{3}$ T. Morimoto,${ }^{4}$ Y. Sun, ${ }^{5}$ J. E. Moore, ${ }^{6}$ and A. G. Grushin ${ }^{7}$ \\ ${ }^{1}$ Donostia International Physics Center, P. Manuel de Lardizabal 4, 20018 Donostia-San Sebastian, Spain \\ ${ }^{2}$ IKERBASQUE, Basque Foundation for Science, Maria Diaz de Haro 3, 48013 Bilbao, Spain \\ ${ }^{3}$ Department of Physics, Massachusetts Institute of Technology, Cambridge, Massachusetts 02139, USA \\ ${ }^{4}$ Department of Applied Physics, The University of Tokyo, Tokyo, 113-8656, Japan \\ ${ }^{5}$ Max Planck Institute for Chemical Physics of Solids, 01187 Dresden, Germany \\ ${ }^{6}$ Department of Physics, University of California, Berkeley, California 94720, USA \\ ${ }^{7}$ Institut Neél, CNRS and Université Grenoble Alpes, Grenoble, France
}

(Received 28 August 2019; published 15 January 2020)

\begin{abstract}
When two lasers are applied to a noncentrosymmetric material, it can generate light at the difference of the incoming frequencies $\Delta \omega$, a phenomenon known as difference frequency generation (DFG), well characterized in semiconductors. In this work, we derive a general expression for DFG in metals, which we use to show that the DFG in chiral topological semimetals under circular polarized light is quantized in units of $e^{3} / h^{2}$ and independent of material parameters, including the scattering time $\tau$, when $\Delta \omega \gg \tau^{-1}$. In this regime, DFG provides a simpler alternative to measure a quantized response in metals compared to previous proposals based on single frequency experiments. Our general derivation unmasks, in addition, a free-carrier contribution to the circular DFG beyond the semiclassical one. This contribution can be written as a Fermi surface integral, features strong frequency dependence, and oscillates with a $\pi / 2$ shift with respect to the quantized contribution. We make predictions for the circular DFG of chiral and nonchiral materials using generic effective models, and $a b$ initio calculations for TaAs and RhSi. Our work provides a complete picture of the DFG in the length gauge approach, in the clean, noninteracting limit, and highlights a plausible experiment to measure topologically quantized photocurrents in metals.
\end{abstract}

DOI: 10.1103/PhysRevResearch.2.012017

One of the most striking predictions that follow from the protected point-like band crossings in topological semimetals is the first quantized observable defined for a metal. It is a circular photocurrent that grows in time at a universal rate given by fundamental constants only in chiral topological semimetals [1,2], which lack mirror symmetries [3-5]. This steadily growing current, known as injection current, originates from resonant, interband transitions and is proportional to the intensity of light. Its universal rate of growth, given by the trace of the circular photogalvanic tensor $\beta^{a b}$, cannot be directly extracted from a steady-state experiment because at times longer than the scattering time $t \gg \tau$, the current saturates to a $\tau$-dependent value, which makes the measurement of the quantized response challenging.

To measure the intrinsic quantized current rate it is rather desirable to use time-dependent electric fields in the form of light pulses of duration shorter than $\tau$, and measure the emitted $\mathrm{THz}$ fields. However, typical experiments are performed in the opposite regime [6-8], where assumptions on the nature of scattering are required to extract the injection current [9].

Published by the American Physical Society under the terms of the Creative Commons Attribution 4.0 International license. Further distribution of this work must maintain attribution to the author(s) and the published article's title, journal citation, and DOI.
In this work, we propose an alternative to access the quantized current rate, which is to measure difference frequency generation (DFG), where two monochromatic light beams of frequencies $\omega \pm \Delta \omega / 2$ produce a slowly oscillating current of frequency $\Delta \omega$. In the limit $\Delta \omega \ll \omega$, DFG is formally equivalent to a photogalvanic effect, but if in addition we demand that $\Delta \omega \gg \tau^{-1}$, the response is intrinsic and $\tau$ independent, exposing the universal quantum.

To show this, we calculate the circular DFG response for all metals in the regime $\omega \gg \Delta \omega \gg \tau^{-1}$, using the length gauge formalism [10-14]. We find that the interband contribution to circular DFG oscillates exactly out of phase with respect to the incoming light, and is given by the intrinsic injection rate $\beta^{a b}$, becoming topologically quantized and independent of material parameters in chiral topological metals. In addition, we find that there is a free-carrier contribution to circular DFG [15] for any metal which oscillates in phase with the incoming light and displays strong $\omega$ dependence due to an extra term beyond the semiclassical Berry dipole [16]. We obtain our results using realistic models for topological semimetals as well as ab initio calculations. Our work contributes to elucidate how topological properties, responsible for unusual nonlinear effects in topological semimetals [17-27], determine nonmonochromatic responses.

Difference frequency generation in metals. DFG is the current response obtained when shining two monochromatic beams $\mathcal{E}_{i}^{a}(t)=\operatorname{Re}\left[E_{i}^{a} e^{-i \omega_{i} t}\right]$ with $i=1,2$ with average frequency $\omega=\left(\omega_{1}+\omega_{2}\right) / 2$ and difference $\Delta \omega=\omega_{1}-\omega_{2}$. 
For concreteness, we consider equal polarizations $E_{1}^{a}=$ $E_{2}^{a}=E^{a}$, and assume time-reversal symmetry throughout the manuscript (we consider the general case in Ref. [28]). In the mentioned regime $\omega \gg \Delta \omega \gg \tau^{-1}$, the generated DFG current can be expanded in perturbation theory in $\Delta \omega / \omega$ as

$$
\begin{aligned}
J^{a}(t)= & 4\left[\frac{\sin (\Delta \omega t)}{\Delta \omega} \beta^{a b}(\omega)+\cos (\Delta \omega t) \gamma^{a b}(\omega)\right]\left[\vec{E} \times \vec{E}^{*}\right]^{b} \\
& +2 \cos (\Delta \omega t) \sigma^{a b c}(\omega)\left(E^{b} E^{c *}+E^{c} E^{b *}\right) .
\end{aligned}
$$

Here we focus on the circular DFG contributions $\beta^{a b}$ and $\gamma^{a b}$ which, unlike $\sigma^{a b c}$ (given in [28]), lead to currents which change sign when the helicity of circularly polarized light is reversed. Explicitly they are

$$
\begin{gathered}
\beta^{a b}(\omega)=\frac{i \pi C}{4} \int_{k} \sum_{n>m} f_{n m} \Delta_{m n}^{a} \operatorname{Im}\left[r_{n m}^{d} r_{m n}^{c}\right] \epsilon^{b c d} \delta\left(\omega_{m n}-\omega\right), \\
\gamma_{1}^{a b}(\omega)=\frac{i C}{2} \int_{k} \sum_{n>m} \frac{\omega f_{n m, a}}{\omega_{n m}^{2}-\omega^{2}} \operatorname{Im}\left[\epsilon^{b c d} r_{n m}^{d} r_{m n}^{c}\right], \\
\gamma_{2}^{a b}(\omega)=-\frac{i C}{2 \omega} \int_{k} \sum_{n}\left[\left(f_{n, a} \Omega_{n}^{b}-\delta^{a b} f_{n, c} \Omega_{n}^{c}\right)\right],
\end{gathered}
$$

where we defined $\gamma^{a b}=\gamma_{1}^{a b}+\gamma_{2}^{a b}$ and assumed $\omega>0$. Here, $C=e^{3} / \hbar^{2}, \int_{k}=\int d^{3} k /(2 \pi)^{3}, f_{n}$ is the Fermi function taken at zero temperature, $f_{n m}=f_{n}-f_{m}$, the Bloch eigenstates are $H|n\rangle=\hbar \omega_{n}|n\rangle, \omega_{n m}=\omega_{n}-\omega_{m}, \Delta_{n m}^{a}=\partial_{k_{a}} \omega_{n m}$, $\xi_{n n}^{a}=i\left\langle n \mid \partial_{k_{a}} n\right\rangle$ is the diagonal Berry connection, and $r_{n m}^{a}=$ $i\left\langle n \mid \partial_{k_{a}} m\right\rangle$ is the interband position matrix element, which is zero unless $n \neq m$. We represent derivatives with a comma, as in $f_{n, a}=\partial_{k_{a}} f_{n}$, while a semicolon denotes the generalized derivative, as in $r_{n m ; a}^{b}=\partial_{k_{a}} r_{n m}^{b}-i\left(\xi_{n n}^{a}-\xi_{m m}^{a}\right) r_{n m}^{b}$. The Berry curvature is $\Omega^{a}=\epsilon^{a b c} \xi_{n n, b}^{c}$.

The tensors $\beta^{a b}$ and $\sigma^{a b c}$ in Eq. (1) are of interband origin and are the DFG analogs of the monochromatic photogalvanic effects known as injection and shift currents, respectively. The tensor $\gamma^{a b}$ is a free-carrier, intraband contribution only present in metals.

The interband contribution to the circular DFG, $\beta^{a b}$, has the same functional form as the interband circular photogalvanic effect. As anticipated, this implies that the trace of $\beta^{a b}$, and thus the corresponding DFG contribution (1), will be quantized in terms of fundamental constants in chiral topological semimetals $[1,2]$.

The free-carrier contribution to the circular DFG, $\gamma^{a b}$, is closely related to the optical Hall conductivity $\sigma_{\text {Hall }}^{a b}(\omega)$ [29]. As we show in Ref. [28], $\gamma^{a d} \epsilon^{d b c}$ can be obtained from $\sigma_{\text {Hall }}^{b c}(\omega)$ by replacing $f_{n} \rightarrow f_{n, a} / \omega$ in its integrand, which explicitly reveals its metallic origin [15].

Within $\gamma^{a b}$ the free-carrier part $\gamma_{2}^{a b}$ given by Eq. (4), is the well studied Berry-dipole term of semiclassical origin and a Fermi surface property [16,30-35]. The novel contribution we report is $\gamma_{1}^{a b}$ in Eq. (3), which is also written as Fermi surface integral. Note that its integrand depends on all bands so $\gamma_{1}^{a b}$ is not a Fermi surface property. Unlike the $1 / \omega$ dependence of the semiclassical contribution, $\gamma_{1}^{a b}$ displays a strong $\omega$ dependence away from zero frequency. At every frequency where a new interband transition becomes active, the energy denominator in Eq. (3) vanishes and the response diverges.
Interestingly, $\gamma_{1}^{a b}$ can also be written in terms of KramersKronig transforms of resonant contributions, a useful property to calculate $\gamma_{1}^{a b}$ using ab initio calculations (see Ref. [28]).

DFG of a single Weyl node. As the simplest example, we first present the circular DFG tensors for a general Weyl semimetal node of the form

$$
H=\sigma_{i} v_{i j} k_{j}+u_{i} k_{i},
$$

written in terms of the Fermi velocity matrix $v_{i j}$, and its tilt $u_{i}$ in the crystallographic coordinate system. For this section, we take $\hbar=1$. We present the detailed computation of the tensors $\beta^{a b}, \gamma_{1}^{a b}, \gamma_{2}^{a b}$ in Ref. [28] and expressed their final form in terms of trace and trace-free parts as

$$
\beta^{a b}=\chi\left(\frac{\delta^{a b}}{3} \beta+\left[\frac{\delta^{a b}}{3}-v_{a c} v_{b d}^{-1} \frac{\tilde{u}_{c} \tilde{u}_{d}}{\tilde{u}^{2}}\right] \beta_{F}\right),
$$

where $\chi=\frac{\operatorname{det} v}{|\operatorname{det} v|}= \pm 1$ is the chirality of the node, $v_{i j}^{-1} \equiv$ $\left(v^{-1}\right)_{i j}$ and $\tilde{u}_{i}=v_{i j}^{-1} u_{j}, \tilde{u}=\sqrt{\tilde{u}_{i}^{2}}$, and similar expressions hold for $\gamma_{1}^{a b}$ and $\gamma_{2}^{a b}$. In terms of the universal photogalvanic constant $\beta_{0} \equiv i C / 4 \pi$ (for reference $\beta_{0}=i \pi e^{3} / h^{2}$ when $\hbar$ is restored) the trace parts are

$$
\begin{gathered}
\beta=-\frac{1}{8} \beta_{0}\left(1-g_{1}(\omega)\right), \\
\gamma_{1}=-\frac{\beta_{0}}{2 \pi} \frac{-\tilde{u} \omega+\mu g_{2}(\omega)}{\tilde{u} \omega^{2}}, \quad \gamma_{2}=-\frac{\beta_{0}}{\pi \omega},
\end{gathered}
$$

and the trace-free parts are

$$
\begin{gathered}
\beta_{F}=\frac{1}{8} \beta_{0} g_{1}(\omega)\left(1-\left[g_{1}(\omega)\right]^{2}\right) \\
\gamma_{1, F}=-\frac{\beta_{0}}{8 \pi} \frac{1}{\tilde{u}^{3} \omega^{2}}\left[\left(4 \tilde{u}^{3}-6 \tilde{u}\right) \omega^{2}-\left(4 \tilde{u}^{2}-12\right) \mu \omega g_{2}(\omega)\right. \\
\left.+3\left(4 \mu^{2}+\left(1-\tilde{u}^{2}\right) \omega^{2}\right) g_{3}(\omega)\right] \\
\gamma_{2, F}=-\frac{\beta_{0}}{4 \pi \omega \tilde{u}^{3}}\left[2 \tilde{u}^{3}-3\left(\tilde{u}+\left(\tilde{u}^{2}-1\right) \operatorname{arctanh}(\tilde{u})\right]\right.
\end{gathered}
$$

where $g_{1}(\omega)=a \Theta(1+a) \Theta(1-a)+\Theta(a-1)-\Theta(-a-$ 1) with $a=\left(\frac{2 \mu}{\omega}-1\right) / \tilde{u}, g_{2}(\omega)=\operatorname{arctanh}\left(\frac{4 \tilde{u} \mu \omega}{4 \mu^{2}+\left(\tilde{u}^{2}-1\right) \omega^{2}}\right)$ and $g_{3}(\omega)=\operatorname{arctanh}\left(\frac{2 \tilde{u} \omega^{2}}{-4 \mu^{2}+\left(\tilde{u}^{2}+1\right) \omega^{2}}\right)$.

The trace $(\beta)$ and traceless $\left(\beta_{F}\right)$ parts are plotted in Figs. 1(a) and 1(b) for zero and finite tilt. As shown in Ref. [1], $\beta$ displays a quantized plateau once the resonant manifold of optical transitions becomes closed, because it is then determined by the monopole charge of the node. In the tilted case, $\tilde{u} \neq 0$ this manifold is open for $2 \mu /(1+\tilde{u})<$ $\omega<2 \mu /(1-\tilde{u})$, and $\beta$ becomes quantized for $\omega>2 \mu /(1-$ $\tilde{u}) . \beta_{F}$ is finite only for $2 \mu /(1+\tilde{u})<\omega<2 \mu /(1-\tilde{u})$, and vanishes in the zero tilt limit. Off-diagonal components of $\beta^{a b}$ therefore require finite tilt [36-38].

The free-carrier trace $\left(\gamma=\gamma_{1}+\gamma_{2}\right)$ and traceless $\left(\gamma_{F}=\right.$ $\left.\gamma_{1, F}+\gamma_{2, F}\right)$ parts are shown in Figs. 1(c) and 1(d). In the zero tilt limit, $\gamma_{1} \propto \omega /\left(4 \mu^{2}-\omega^{2}\right)$. At finite tilt, the divergence at $2 \mu$ splits into two logarithmic divergences at $\omega_{ \pm}=2 \mu /(1 \pm$ $\tilde{u})$, where $\gamma_{1, F}$ also displays strong singularities. We note that $\gamma_{1}^{a b}$ is well behaved when $\omega \rightarrow 0$. The singularities at $\omega \rightarrow 0$ originate from $\gamma_{2}$ and $\gamma_{2, F}$. While the trace of the semiclassical free-carrier contribution $\gamma_{2}$ gives a universal $1 / \omega$ divergence that is independent of the chemical potential and tilt [34], $\gamma_{2, F}$ 

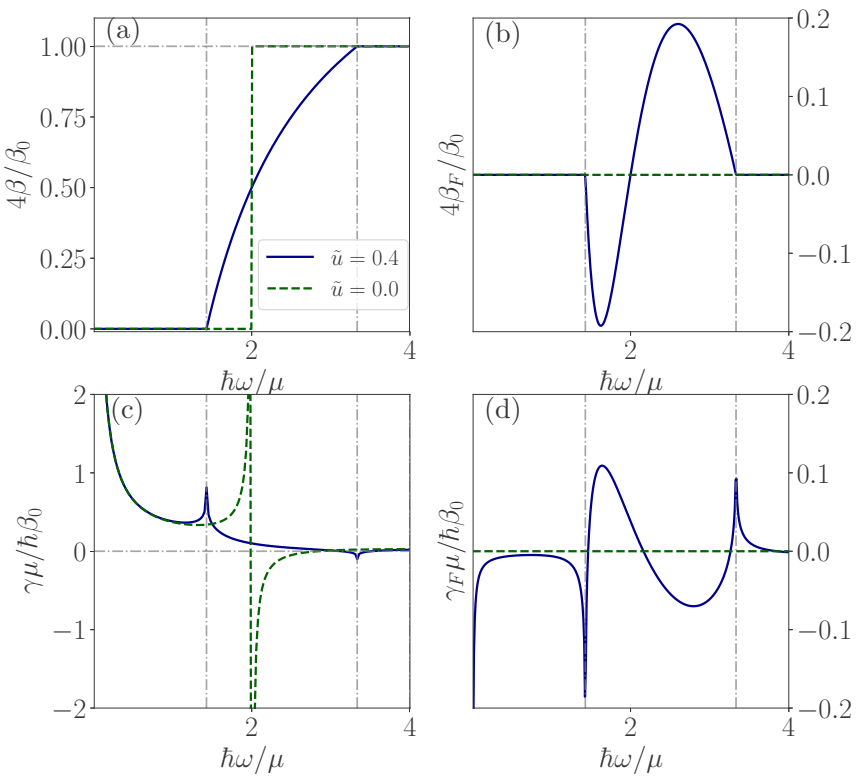

FIG. 1. Trace and traceless parts of CPGE tensors for a Weyl node with chirality $\chi=-1$, with tilt $\tilde{u}=0$ (green dashed) and $\tilde{u}=$ 0.4 (blue solid). (a) Trace of injection tensor $\beta$, where a quantized plateau is observed. (b) Traceless part of injection $\beta_{F}$. (c) Trace of free-carrier tensor $\gamma$. Note the $1 / \omega$ divergence stemming from $\gamma_{2}$ which always cancels after summing over all nodes in the Brillouin zone. (d) Traceless part of free-carrier $\gamma_{F}$. (a), (b) and (c), (d) are in units of $\beta_{0}=i \pi e^{3} / h^{2}$ and $\beta_{0} / \mu$, respectively. Vertical dashed lines mark the frequencies $\hbar \omega_{ \pm}=2 \mu /(1 \pm \tilde{u})$.

displays a nonuniversal $1 / \omega$ divergence, which depends on the tilt. Since in any material the number of left and right chiralities must be equal, the sum over Weyl nodes will cancel all the trace contributions $\gamma_{2}$ in pairs. The total free-carrier current, however, may have a nonuniversal $1 / \omega$ pole due to $\gamma_{2, F}$ because of its tilt dependence and the tensor structure in Eq. (6).

$D F G$ quantization in chiral topological semimetals. For the trace $\beta$ to be quantized, a topological semimetal is required to have left and right chirality nodes at different energies [1,2], which is only allowed in a chiral lattice structure, which lacks mirror symmetries.

Consider first two Weyl nodes of opposite chiralities away from time-reversal invariant points located at energies $\mu_{L}$ and $\mu_{R}$ measured from the chemical potential and tilts $\tilde{u}_{L}$ and $\tilde{u}_{R}$ (e.g., $\mathrm{SrSi}_{2}$ without spin-orbit coupling [4]). In the presence of time-reversal symmetry, two more symmetry related nodes exist, which contribute in exactly the same way and simply double the result we present. In Figs. 2(a) and 2(b), we show the circular DFG trace parts $\beta$ and $\gamma$. The quantized plateau seen for $\beta$ is realized in the range $2 \mu_{L} /\left(1-\tilde{u}_{L}\right)<$ $\omega<2 \mu_{R} /\left(1+\tilde{u}_{R}\right)$, which determines how large the tilts can be before quantization is lost. Note also that, upon summing over the two chiralities, the total trace part $\gamma$ presents no $1 / \omega$ pole, as discussed above.

Our second example are multifold fermions. They are low energy excitations close to degeneracy nodes where three, four, or six bands meet, and which requires additional crystalline symmetries to remain degenerate [39-43]. They have a definite chirality and larger monopole charge compared to
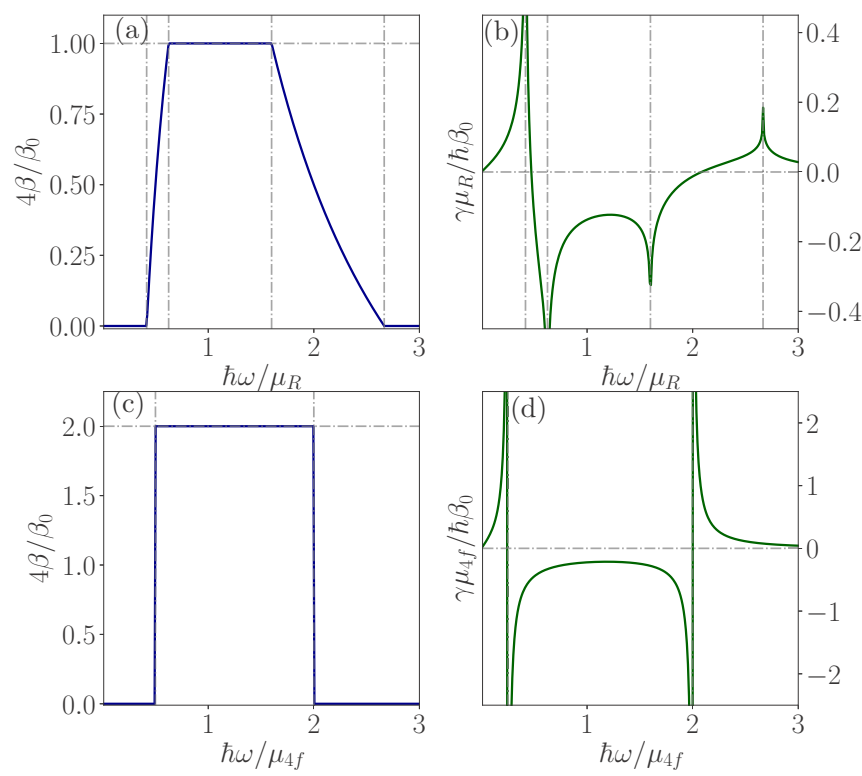

FIG. 2. [(a) and (b)] Diagonal parts of the circular DFG, $\beta$ (blue) and $\gamma$ (green) for a chiral Weyl semimetal with two Weyl nodes at energies $\mu_{L}$ and $\mu_{R}$, with $\mu_{L} / \mu_{R}=0.25$, and tilts $\tilde{u}_{L}=0.2 \tilde{u}_{R}=$ 0.25 . [(c) and (d)] Same quantities for the linear approximation of a multifold material in space group 198, with a threefold at $\mu_{3 f}$ and a double Weyl fourfold fermion at $\mu_{4 f}$ with $\mu_{3 f} / \mu_{4 f}=0.25$.

Weyl nodes. In space group 198, to linear order in momentum and neglecting spin-orbit coupling, a threefold fermion exists at the $\Gamma$ point with a Hamiltonian $H=v_{F} \mathbf{k} \cdot \mathbf{S}$, where $\mathbf{S}$ is a vector of three spin-1 matrices. At the $R$ point, a fourfold fermion exists composed of two Weyl nodes of equal chiralities, $H=-v_{F} \mathbf{k} \cdot \sigma \otimes 1$, separated in energy from the threefold at $\Gamma$. This situation is realized in CoSi [44-46], $\mathrm{RhSi}$ [46], and AlPt [47], which motivate our example.

In Figs. 2(c) and 2(d), we show the circular DFG trace parts $\beta$ and $\gamma$ corresponding to a threefold and a fourfold nodes at energies $\mu_{3 f}$ and $\mu_{4 f}$ (see Ref. [28] for analytic expressions). Due to the monopole charge carried by the multifold fermions [2], $\beta$ displays a quantized plateau at $\beta_{0} / 2$, two times that of a Weyl node. The free-carrier part $\gamma$ displays divergences at every energy where a new band becomes resonant with the Fermi level. As for the chiral Weyl case, upon summing over chiralities the low-energy divergent semiclassical part is absent. The remaining free-carrier contribution is $\gamma_{1}=$ $\left(\beta_{0} / \pi\right) \sum_{i} \chi_{i} \omega /\left(\mu_{i}^{2}-\omega^{2}\right)$ where $\mu_{i}=\mu_{3 f}, 2 \mu_{4 f}$ and $\chi_{i}=$ \pm . This is a universal function for linear nodal points occurring at time-reversal invariant momenta.

Similarly to multifods, Kramers Weyl semimetals [5] like $\mathrm{TlTe}_{2} \mathrm{O}_{6}$ present Weyl nodes at time-reversal invariant momenta separated in energy. The full DFG tensor can be calculated analytically, and it is detailed in Ref. [28] since it is conceptually similar to our previous examples.

DFG in TaAs and RhSi from first principles. In the presence of mirror symmetries the trace of $\gamma^{a b}$ and $\beta^{a b}$ vanish, with the consequent loss of quantization. In a system with $C_{4 v}$ symmetry like TaAs [48-52], the only allowed component is antisymmetric $\beta^{x y}=-\beta^{y x}$, which can only be due to the traceless part in Eq. (6). The same requirement holds for $\gamma^{a b}$. Moreover, from Eq. (6), both tilt and nontrivial $v_{i j}$ are required 

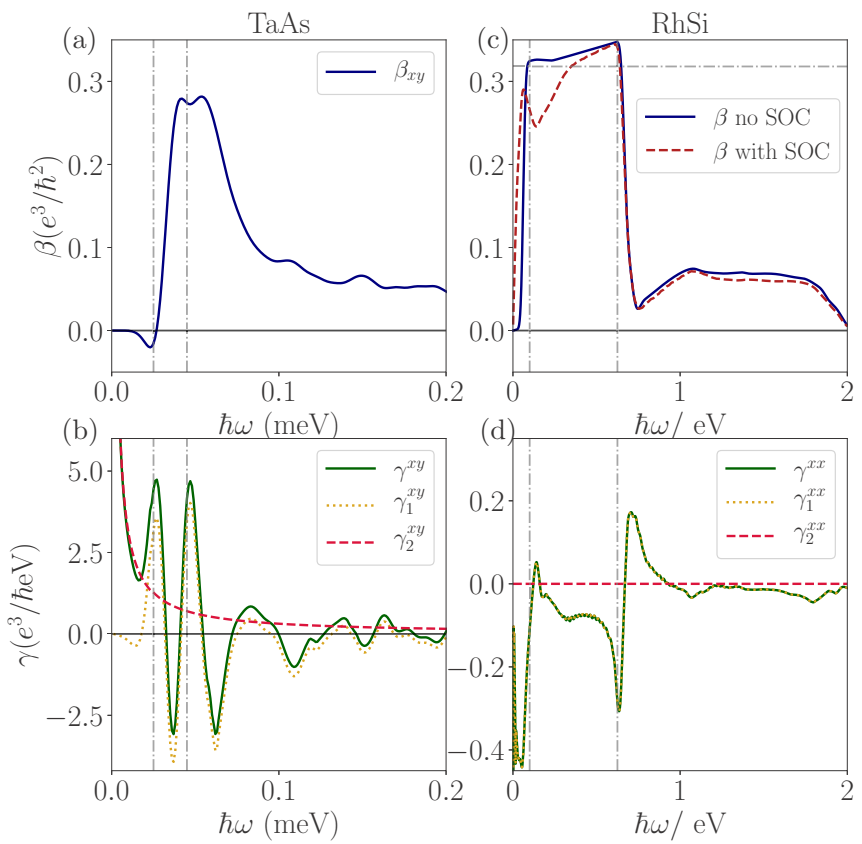

FIG. 3. First-principles prediction for circular DFG. a) and b) show $\beta_{x y}$ and $\gamma_{x y}$ for TaAs with spin-orbit coupling (SOC). The activation frequencies of the $\mathrm{W} 2$ and $\mathrm{W} 1$ pairs of nodes at 25 and $45 \mathrm{meV}$ (dashed vertical lines) mark the peaks in (a) and (b), in qualitative agreement with Figs. 1(b) and 1(d). In (c) and (d), we show the trace $\beta$ (with and without SOC) and $\gamma_{x x}$ with SOC for RhSi. The activation frequencies of the threefold at $\Gamma$ and fourfold fermion at $R, 0.1$ and $0.625 \mathrm{eV}$, respectively, are marked by dashed vertical lines. The horizontal dashed line in (b) marks the effective monopole charge of 4 corresponding to $4 \beta_{0}=0.318 e^{3} / \hbar^{2}$. For (b) and (d), the total free-carrier contribution $\gamma$ (green solid) is composed of the semiclassical $\left(\gamma_{2}\right.$, red dashed) and the novel free-carrier contribution ( $\gamma_{1}$, orange dotted), which qualitatively agree with Fig. 2(d).

to produce a finite result in TaAs. If $v_{i j}=\delta_{i j}$ this component must also be zero $[38,53]$, regardless of the tilt, since Eq. (6) becomes symmetric.

In order to provide a qualitative prediction, we have calculated the different parts of the circular DFG for TaAs using density functional theory (DFT) (see Ref. [28] for details). The results are shown in Figs. 3(a) and 3(b). We observe that $\gamma^{y x}$ shows the $1 / \omega$ divergence, and several sign changes close to $2 \mu_{W_{i}}$, where $\mu_{W_{i}}$ is the energy of the two types of Weyl nodes W1 and W2 present in TaAs $[48,49,54]$. Consistently, $\beta^{y x}$ exhibits characteristic peaks around $2 \mu_{W_{i}}$. These features follow qualitatively those expected for the traceless circular DFG components, shown in Figs. 3(b) and 3(d).

Unlike TaAs, materials in the cubic space group 198, such as $\mathrm{RhSi}$, CoSi or AlPt, are chiral and lack mirror symmetry. Taking RhSi as an example and using cubic symmetry, we have calculated the diagonal components of the DFG using DFT [see Figs. 1(c) and 1(d)]. Consistent with Ref. [2] we find that $\beta$ displays a flat region between the activation frequencies of the threefold and fourfold fermions. However the response is not exactly quantized: it is corrected by the presence of additional bands deviating from exact quantization even in the absence of spin-orbit coupling. These corrections are expected to decrease upon decreasing the chemical potential. Lastly, the free-carrier contribution $\gamma_{x x}$ shows peaks and sign changes close to the activation frequencies of the threefold and fourfold fermions, consistent with Fig. 2(d).

Discussion. In this work, we have found that the circular DFG response in the clean limit is composed of two contributions that oscillate out of phase, $\beta^{a b}$ and $\gamma^{a b}$ : the trace of the former is quantized in chiral topological metals, and the latter is a free-carrier contribution that vanishes in the absence of a Fermi surface.

The measurement of the quantized trace of $\beta^{a b}$ can be achieved if the hierarchy of scales $\omega \gg \Delta \omega \gg \tau^{-1}$ is met. Interestingly, evaluating if this condition is met is possible without prior knowledge of $\tau$ : when $\Delta \omega \gg \tau^{-1}$ the interband circular DFG signal oscillates exactly out of phase with the incoming light, while in the dirty limit $\Delta \omega \ll \tau^{-1}$, it oscillates in phase. Similarly, when $\Delta \omega \gg \tau^{-1}$ the free-carrier circular DFG $\gamma^{a b}$ oscillates in-phase and displays a characteristic singular $\omega$ dependence. The observation of an out-of-phase smooth component together with an in-phase component with singularities is therefore a strong signal that the clean limit has been achieved.

In the currently available $\mathrm{RhSi}$, the plateau extends up to $\hbar \omega \sim 0.7 \mathrm{eV}$ [9], so setting $\Delta \omega=100 \mathrm{meV}$ may allow scattering rates as large as $\tau^{-1} \sim 20 \mathrm{meV}$ or $\tau \sim 200 \mathrm{fs}$. It should be noted that the quantized plateau is corrected in multifold materials by multi-band corrections [2], as confirmed by our DFT calculations, so it is desirable to search for new chiral Weyl semimetals with simpler band structures to reach exact quantization.

The relation of the free-carrier DFG response with the more often measured DC photogalvanic responses (where $\Delta \omega=0$ with finite $\tau$ ) is quite subtle. Both general arguments and explicit calculations have been used to argue that the freecarrier part of the DC photocurrent vanishes in the absence of a specific mechanism for dissipation [55-57]. In the dirty limit $\left(\Delta \omega \ll \tau^{-1}\right)$, there are $\tau$-independent disorder-induced contributions which can cancel the apparently intrinsic contributions [55], in a situation reminiscent of the anomalous Hall effect. In view of other disorder-induced photocurrent calculations [37,58-61], the extent to which this cancellation happens remains to be understood. Due to our assumption that $\Delta \omega \gg \tau^{-1}$ the above subtleties do not affect the DFG regime pertinent to this work.

In conclusion, our work shows how difference frequency generation provides a disorder-independent route to separately measure photocurrent quantization, and a novel Fermi surface contribution beyond semiclassics.

Acknowledgments. We are grateful to B. Yan and L. Wu for enlightening discussions, and to L. Golub and M. Teixido for providing us with copies of Ref. [55]. We acknowledge O. Matsyshyn and I. Sodemann for valuable discussions, and for sharing their manuscript on a study employing a similar formalism prior to publication [62]. A.G.G. acknowledges funding from the European Union's Horizon 2020 research and innovation programme under the Marie-Sklodowska Curie Grant agreement No. 653846 and the ANR under the grant ANR-18-CE30-0001-01. J.E.M. was supported by the Quantum Materials program at LBNL, funded by the US Department of Energy under Contract No. DE-AC02-05CH11231. 
[1] F. de Juan, A. Grushin, T. Morimoto, and J. Moore, Nat. Commun. 8, 15995 (2017).

[2] F. Flicker, F. de Juan, B. Bradlyn, T. Morimoto, M. G. Vergniory, and A. G. Grushin, Phys. Rev. B 98, 155145 (2018).

[3] M. Hirayama, R. Okugawa, S. Ishibashi, S. Murakami, and T. Miyake, Phys. Rev. Lett. 114, 206401 (2015).

[4] S.-M. Huang, S.-Y. Xu, I. Belopolski, C.-C. Lee, G. Chang, T.-R. Chang, B. Wang, N. Alidoust, G. Bian, M. Neupane, D. Sanchez, H. Zheng, H.-T. Jeng, A. Bansil, T. Neupert, H. Lin, and M. Z. Hasan, Proc. Natl. Acad. Sci. USA 113, 1180 (2016).

[5] G. Chang, B. J. Wieder, F. Schindler, D. S. Sanchez, I. Belopolski, S.-M. Huang, B. Singh, D. Wu, T.-R. Chang, T. Neupert, S.-Y. Xu, H. Lin, and M. Z. Hasan, Nat. Mater. 17, 978 (2018).

[6] N. Laman, M. Bieler, and H. Van Driel, J. Appl. Phys. 98, 103507 (2005).

[7] M. Bieler, K. Pierz, and U. Siegner, J. App. Phys. 100, 083710 (2006).

[8] D. A. Bas, R. A. Muniz, S. Babakiray, D. Lederman, J. Sipe, and A. D. Bristow, Opt. Express 24, 23583 (2016).

[9] D. Rees, K. Manna, B. Lu, T. Morimoto, H. Borrmann, C. Felser, J. Moore, D. H. Torchinsky, and J. Orenstein, arXiv:1902.03230.

[10] J. E. Sipe and E. Ghahramani, Phys. Rev. B 48, 11705 (1993).

[11] C. Aversa and J. E. Sipe, Phys. Rev. B 52, 14636 (1995).

[12] J. E. Sipe and A. I. Shkrebtii, Phys. Rev. B 61, 5337 (2000).

[13] F. Nastos and J. E. Sipe, Phys. Rev. B 74, 035201 (2006).

[14] F. Nastos and J. E. Sipe, Phys. Rev. B 82, 235204 (2010).

[15] V. Genkin and P. Mednis, Sov. Phys. JETP 27, 609 (1968).

[16] I. Sodemann and L. Fu, Phys. Rev. Lett. 115, 216806 (2015).

[17] X. Yang, K. Burch, and Y. Ran, arXiv:1712.09363.

[18] Y. Zhang, H. Ishizuka, J. van den Brink, C. Felser, B. Yan, and N. Nagaosa, Phys. Rev. B 97, 241118(R) (2018).

[19] Q. Ma, S.-Y. Xu, C.-K. Chan, C.-L. Zhang, G. Chang, Y. Lin, W. Xie, T. Palacios, H. Lin, S. Jia et al., Nat. Phys. 13, 842 (2017).

[20] G. B. Osterhoudt, L. K. Diebel, M. J. Gray, X. Yang, J. Stanco, X. Huang, B. Shen, N. Ni, P. J. W. Moll, Y. Ran, and K. S. Burch, Nat. Mater. 18, 471 (2019).

[21] L. Wu, S. Patankar, T. Morimoto, N. L. Nair, E. Thewalt, A. Little, J. G. Analytis, J. E. Moore, and J. Orenstein, Nat. Phys. 13, 350 (2017).

[22] S. Patankar, L. Wu, B. Lu, M. Rai, J. D. Tran, T. Morimoto, D. E. Parker, A. G. Grushin, N. L. Nair, J. G. Analytis, J. E. Moore, J. Orenstein, and D. H. Torchinsky, Phys. Rev. B 98, 165113 (2018).

[23] Z. Ji, G. Liu, Z. Addison, W. Liu, P. Yu, H. Gao, Z. Liu, A. M. Rappe, C. L. Kane, E. J. Mele et al., Nat. Mater. 18, 955 (2019).

[24] P. Hosur, Phys. Rev. B 83, 035309 (2011).

[25] T. Morimoto and N. Nagaosa, Sci. Adv. 2, e1501524 (2016).

[26] D. E. Parker, T. Morimoto, J. Orenstein, and J. E. Moore, Phys. Rev. B 99, 045121 (2019).

[27] E. J. König, M. Dzero, A. Levchenko, and D. A. Pesin, Phys. Rev. B 99, 155404 (2019).

[28] See Supplemental Material at http://link.aps.org/supplemental/ 10.1103/PhysRevResearch.2.012017 for the full derivation of the DFG response function, the analytical calculations for model Hamiltonians, and the details of the ab initio calculations.

[29] J. F. Steiner, A. V. Andreev, and D. A. Pesin, Phys. Rev. Lett. 119, 036601 (2017).
[30] E. Deyo, L. Golub, E. Ivchenko, and B. Spivak, arXiv:0904.1917.

[31] J. E. Moore and J. Orenstein, Phys. Rev. Lett. 105, 026805 (2010).

[32] T. Morimoto, S. Zhong, J. Orenstein, and J. E. Moore, Phys. Rev. B 94, 245121 (2016).

[33] H. Ishizuka, T. Hayata, M. Ueda, and N. Nagaosa, Phys. Rev. B 95, 245211 (2017).

[34] H. Rostami and M. Polini, Phys. Rev. B 97, 195151 (2018).

[35] Y. Zhang, Y. Sun, and B. Yan, Phys. Rev. B 97, 041101(R) (2018).

[36] C.-K. Chan, N. H. Lindner, G. Refael, and P. A. Lee, Phys. Rev. B 95, 041104(R) (2017).

[37] E. J. König, H.-Y. Xie, D. A. Pesin, and A. Levchenko, Phys. Rev. B 96, 075123 (2017).

[38] L. Golub, E. L. Ivchenko, and B. Spivak, JETP Lett. 105, 782 (2017).

[39] J. L. Mañes, Phys. Rev. B 85, 155118 (2012).

[40] B. Bradlyn, J. Cano, Z. Wang, M. G. Vergniory, C. Felser, R. J. Cava, and B. A. Bernevig, Science 353, aaf5037 (2016).

[41] G. Chang, S.-Y. Xu, B. J. Wieder, D. S. Sanchez, S.-M. Huang, I. Belopolski, T.-R. Chang, S. Zhang, A. Bansil, H. Lin, and M. Z. Hasan, Phys. Rev. Lett. 119, 206401 (2017).

[42] P. Tang, Q. Zhou, and S.-C. Zhang, Phys. Rev. Lett. 119, 206402 (2017).

[43] A. Bouhon and A. M. Black-Schaffer, Phys. Rev. B 95, 241101(R) (2017).

[44] D. Takane, Z. Wang, S. Souma, K. Nakayama, T. Nakamura, H. Oinuma, Y. Nakata, H. Iwasawa, C. Cacho, T. Kim, K. Horiba, H. Kumigashira, T. Takahashi, Y. Ando, and T. Sato, Phys. Rev. Lett. 122, 076402 (2019).

[45] Z. Rao, H. Li, T. Zhang, S. Tian, C. Li, B. Fu, C. Tang, L. Wang, Z. Li, W. Fan, J. Li, Y. Huang, Z. Liu, Y. Long, C. Fang, H. Weng, Y. Shi, H. Lei, Y. Sun, T. Qian, and H. Ding, Nature (London) 567, 496 (2019).

[46] D. S. Sanchez, I. Belopolski, T. A. Cochran, X. Xu, J.-X. Yin, G. Chang, W. Xie, K. Manna, V. Süß, C.-Y. Huang, N. Alidoust, D. Multer, S. S. Zhang, N. Shumiya, X. Wang, G.-Q. Wang, T.-R. Chang, C. Felser, S.-Y. Xu, S. Jia, H. Lin, and M. Z. Hasan, Nature (London) 567, 500 (2019).

[47] N. B. M. Schröter, D. Pei, M. G. Vergniory, Y. Sun, K. Manna, F. de Juan, J. A. Krieger, V. Süß, M. Schmidt, P. Dudin, B. Bradlyn, T. K. Kim, T. Schmitt, C. Cacho, C. Felser, V. N. Strocov, and Y. Chen, Nat. Phys. 15, 759 (2019).

[48] H. Weng, C. Fang, Z. Fang, B. A. Bernevig, and X. Dai, Phys. Rev. X 5, 011029 (2015).

[49] S.-M. Huang, S.-Y. Xu, I. Belopolski, C.-C. Lee, G. Chang, B. Wang, N. Alidoust, G. Bian, M. Neupane, C. Zhang, S. Jia, A. Bansil, H. Lin, and M. Z. Hasan, Nat. Commun. 6, 7373 (2015).

[50] B. Q. Lv, N. Xu, H. M. Weng, J. Z. Ma, P. Richard, X. C. Huang, L. X. Zhao, G. F. Chen, C. E. Matt, F. Bisti, V. N. Strocov, J. Mesot, Z. Fang, X. Dai, T. Qian, M. Shi, and H. Ding, Nat. Phys. 11, 724 (2015).

[51] S.-Y. Xu, I. Belopolski, N. Alidoust, M. Neupane, G. Bian, C. Zhang, R. Sankar, G. Chang, Z. Yuan, C.-C. Lee, S.-M. Huang, H. Zheng, J. Ma, D. S. Sanchez, B. Wang, A. Bansil, F. Chou, P. P. Shibayev, H. Lin, S. Jia, and M. Z. Hasan, Science 349, 613 (2015).

[52] L. X. Yang, Z. K. Liu, Y. Sun, H. Peng, H. F. Yang, T. Zhang, B. Zhou, Y. Zhang, Y. F. Guo, M. Rahn, D. Prabhakaran, 
Z. Hussain, S. K. Mo, C. Felser, B. Yan, and Y. L. Chen, Nat. Phys. 11, 728 (2015).

[53] N. Leppenen, E. Ivchenko, and L. Golub, JETP 129, 139 (2019).

[54] J. Buckeridge, D. Jevdokimovs, C. R. A. Catlow, and A. A. Sokol, Phys. Rev. B 93, 125205 (2016).

[55] V. Belinicher, E. Ivchenko, and G. Pikus, Sov. Phys. Semicond. 20, 558 (1986).

[56] V. I. Belinicher and B. I. Sturman, Sov. Phys. Usp. 23, 199 (1980).
[57] V. I. Belinicher, E. L. Ivchenko, and B. I. Sturman, Sov. Phys. JETP 56, 359 (1982).

[58] H. Isobe, S.-Y. Xu, and L. Fu, arXiv:1812.08162.

[59] L. E. Golub and E. L. Ivchenko, Phys. Rev. B 98, 075305 (2018).

[60] S. Nandy and I. Sodemann, Phys. Rev. B 100, 195117 (2019).

[61] Z. Du, C. Wang, S. Li, H.-Z. Lu, and X. Xie, Nat. Commun. 10, 3047 (2019).

[62] O. Matsyshyn and I. Sodemann, Phys. Rev. Lett. 123, 246602 (2019). 